\title{
The birth of a new journal dedicated to infectious diseases
}

\author{
Nigel Silman* \\ Senior Business Development Manager, Public Health England, Porton Down, Salisbury, UK
}

It is with very great pleasure that I welcome you to, this, our first edition of the Journal of Clinical Microbiology \& Infectious Diseases. Of course, this has been in incubation for some time but even allowing for the well-described "lag" phase, to coin a bacterial growth term, growth has been rapid and we have moved from a position of an outline idea in January to the launch of the journal with our first peer-reviewed articles. Naturally this has been a huge team effort with considerable input from our editorial team and the supporting infrastructure provided by the Managing Editor and team at Open Access Text (OAT).

Naturally, we now want to hear from you! Successful journals offer a platform for researchers to share and discuss their findings and we sincerely hope that you will consider publishing your next paper with us in CMID. Not only do we actively seek your submission, but we would also love to hear from you if you would like to be considered for membership of our scientific editorial board. This is a great opportunity to join an experienced editorial team and we would particularly welcome applications from those in the early stages of their careers. Please let me know via our editorial office if you wish to confidentially discuss this opportunity or would like further information.

Well enough of the sales pitch, what about me? I was keen to support the launch of an open-access journal in this subject field due to my long-standing interest and expertise in infectious disease research. It is a quite sobering thought that three of the top ten causes of death worldwide are still infectious diseases; these are lower respiratory infections/pneumonia, diarrhoeal diseases and HIV/AIDS (source WHO, 2012). It is quite staggering that in the $21^{\text {st }}$ century that we still observe such high mortality figures for what are, in at least two of these three causes, preventable and treatable diseases. Indeed, many Governments worldwide have risks due to infectious disease outbreaks at the top or very high on their national risk registers, thus the threat from communicable diseases certainly has not gone away. The recently highlighted crisis around antimicrobial use has also reminded large sections of the worldwide population that previously treatable infections may/are becoming a major problem again. We have also seen two major infectious disease outbreaks, caused by Ebola and Zika viruses respectively. One of these outbreaks is, of course, ongoing and potentially impacts the upcoming Olympic Games in Brazil; such is the widespread effect of communicable diseases. It is clear to me that research in the area of clinical microbiology and infectious diseases has a huge part to play in understanding the basic biology of, in particular, emerging infectious diseases, such that faster, improved interventions may be brought to bear.

Whilst thinking about intervention, one of my key interests is the research, development and use of rapid diagnostic approaches, including point of care and biosensor approaches, to combat infectious disease outbreaks. One of our most effective weapons against spread of communicable disease is to use quarantine methods to prevent person to person transmission where this is a key transmission route. By rapid, accurate diagnosis, we can quickly help to control disease spread by limiting community contact. By the same token, in disease outbreaks, such as the recent Ebola crisis, the deployment of mobile laboratories to outbreak areas was a key control measure in managing the transmission of this blood-borne disease.

I hope you enjoy the papers presented in this first edition. To whet your appetite, we will be bringing you a set of papers describing the set-up, operation and training required to mobilise a laboratory into an outbreak as well as providing reflections on lessons learnt from some of the scientists who actually performed these roles. We hope to bring you these soon in the next edition.
Copyright: (C2016 Silman N. This is an open-access article distributed under the terms of the Creative Commons Attribution License, which permits unrestricted use, distribution, and reproduction in any medium, provided the original author and source are credited.
Correspondence to: Nigel Silman, Senior Business Development Manager, Public Health England, Porton Down, Salisbury, UK, Tel: +44 (0)1980 612863; Mob: +44 (0)7917 558474; Fax: +44 (0)1980 612621; E-mail: nigel.silman@phe.gov.uk

Received: July 04, 2016; Accepted: July 06, 2016; Published: July 09, 2016 